\title{
Understanding UnCERTAinty SHOCKS AND THE Role of BlaCK SWANS
}

\author{
Anna Orlik and Laura Veldkamp ${ }^{1}$
}

February 8, 2014

\footnotetext{
${ }^{1}$ Please send comments to anna.a.orlik@frb.gov and lveldkam@stern.nyu.edu. We are grateful to the seminar participants at Wharton, Yale SOM, Banque de France, Toulouse School of Economics, U. Quebec at Montreal, Conference on Macroeconomic Uncertainty at Dallas Federal Reserve, the NBER Summer Institute, NYU Alumni Conference, North American and European meetings of the Econometric Society, AEA meetings, Becker-Friedman Institute Policy Uncertainty Workshop, Federal Reserve Bank of Boston, the NYU macro lunch, Tom Stark, and our AEA discussant, Lars Hansen. Many thanks also to Isaac Baley, David Johnson, Callum Jones and Nic Kozeniauskas for outstanding research assistance. We acknowledge grant assistance from the Stern Center for Global Economy and Business. The views expressed herein are those of the authors and do not necessarily reflect the position of the Board of Governors of the Federal Reserve or the Federal Reserve System.
} 


\begin{abstract}
A recent literature explores many ways in which uncertainty shocks can have important economic effects. But how large are uncertainty shocks and where do they come from? Researchers typically estimate a model with stochastic volatility, using all available data, then condition on the estimated model to infer volatility. This volatility is the uncertainty of an agent who knows the true probability of outcomes and whose only uncertainty is about what the draw from that distribution will be. We model a Bayesian forecaster who uses new data released each quarter to re-estimate the parameters that govern the shape of the probability distribution of GDP growth. Although the forecaster's parameter revisions are small, the probability of black swans (extreme events) is very sensitive to these revisions. Our real-time measure of GDP forecast uncertainty reveals that changes in the risk of a black swan explain most of the shocks to uncertainty.
\end{abstract}


Some times are uncertain times for the aggregate economy. At other times, events appear to be predictable and confidence is high. An active emerging literature argues that changes in uncertainty can explain asset pricing, banking crises, business cycle fluctuations, and the 2007 financial crisis. But these theories do not explain why people suddenly become uncertain. We examine whether a simple, Bayesian belief formation process can generate large enough fluctuations in uncertainty to explain recessions and financial crises.

One source of uncertainty shocks is volatility shocks. The variance of unpredictable innovations to output could rise. If an agent knows the true law of motion for the economy, then all uncertainty shocks come from changes in volatility. But if an agent does not know the true law of motion of the economy because he does not know the true parameter values that govern the model, then new sources of uncertainty shocks emerge. We estimate two Bayesian forecasting models and compute uncertainty (conditional standard deviation) of the forecast of next quarter's GDP growth, recognizing that since the estimated parameters have standard errors, they are themselves uncertain. In each quarter, we update the forecast and model parameter estimates, using all U.S. GDP growth data that was available at that time.

Acknowledging parameter uncertainty uncovers two new sources of uncertainty shocks. One source of shocks is surprises: events that are improbable under the previous period's estimated model. If an event occurs that a model says is improbable, a Bayesian agent concentrates less probability weight on that model's parameters; standard errors on the parameter estimate often rises and uncertainty increases. Of course, an outlier event also leads a stochastic volatility model to predict higher volatility. That makes these mechanisms hard to distinguish. But more importantly, uncertainty shocks generated from this first mechanism are tiny.

The second source of uncertainty is something we call "black swan risk," which is the conditional probability of a growth realization more negative than any observed so far. When the forecasting model implies a normal distribution of outcomes, the probability of an n-standard-deviation event is constant. But when we allow our forecaster to estimate skewness, the probability of negative outliers can fluctuate. For example, a new piece of data can lead the forecaster to estimate more negative skewness, which makes extreme negative outcomes more likely and thus raises uncertainty. Because the probability of 
tail events is very sensitive to the parameters that regulate skewness, black swan risk is very volatile and generates large changes in uncertainty. When we apply this model to GDP data, we find that most of the shocks to uncertainty are explained by changes in the estimated probability of black swans.

We define macroeconomic uncertainty as the standard deviation of next-period GDP growth $y_{t+1}$, conditional on all information observed through time $t: \operatorname{Std}\left[y_{t+1} \mid \mathcal{I}_{t}\right]$. We use this definition because in most models, this is the theoretically-relevant moment. When there is an option value of waiting, beliefs with a higher conditional variance (imprecise beliefs) raise the value of waiting to observe additional information. Thus, it is uncertainty in the form of a higher conditional variance that typically delays consumption or investment and thus depresses economic activity.

To build up intuition for how estimation uncertainty behaves, we start with one of the simplest forecasting models: a linear model of GDP growth with a continuous hidden state and homoskedastic innovations. We hold the volatility of the innovations fixed so that we can isolate the effect of parameter uncertainty shocks. Each period $t$, our forecaster observes time- $t$ GDP growth and uses the complete history of GDP data to estimate her model and forecast GDP growth in $t+1$. In such a setting, an agent with known model parameters faces no uncertainty shocks because the estimated model is a homoskedastic one. But the forecaster with parameter uncertainty experiences changes in the conditional variance of her forecasts. These are uncertainty shocks.

However, a linear model with normally distributed shocks generates only small uncertainty shocks, those shocks are not counter-cyclical, and its forecasts badly miss a key feature of forecast data: The average forecast is significantly lower than the average GDP growth realization. For an unbiased forecaster with a linear model, this should not be the case. But if the true distribution of GDP growth is not known and is believed to be negatively skewed, we show that expected values can be systematically lower than the realizations. Therefore, we explore a non-linear forecasting model and find that combining non-linear forecasting with parameter uncertainty generates large shocks to uncertainty, particularly in recessions.

We compare our model-based uncertainty series to commonly-used uncertainty proxies and find that it is less variable, but more persistent than the proxy variables. The most 
highly correlated proxies are the price of a volatility option (VIX) and the Baker, Bloom and Davis (2012) uncertainty index.

A key message of our paper is that understanding and measuring economic uncertainty requires relaxing the full-information assumptions of rational expectations econometrics. In econometrics, rational expectations typically describes the assumption that agents know the true law of motion of the economy. In such a world, agents have no uncertainty about what the distribution of economic outcomes is. Their only uncertainty is about what realization will be drawn from that distribution. To measure the uncertainty of such a forecaster, it makes sense to estimate a model on as much data as possible, take the parameters as given, and estimate the conditional standard deviation of model innovations. This is what stochastic volatility estimates typically are. But in reality, the macroeconomy is not governed by a simple, known model and we surely don't know its parameters. Instead, our forecast data (from the Survey of Professional Forecasters or SPF) suggests that forecasters estimate simple models to approximate complex processes and constantly use new data to update beliefs. Forecasters are not irrational. They simply do not know the economy's true data-generating process. In such a setting, uncertainty and volatility can behave quite differently.

Related literature A new and growing literature uses uncertainty shocks as a driving process to explain business cycles (e.g., Bloom, Floetotto, Jaimovich, Sapora-Eksten, and Terry (2012), Basu and Bundick (2012) Christiano, Motto, and Rostagno (2012), Bianchi, Ilut, and Schneider (2012)), to explain investment dynamics Bachmann and Bayer (2012a), to explain asset prices (e.g., Bansal and Shaliastovich (2010), Pastor and Veronesi (2012)), and to explain banking panics (Bruno and Shin, 2012). These papers are complementary to ours. We explain where uncertainty shocks come from, while these papers trace out the economic and financial consequences of the shocks. ${ }^{1}$

A small set of related theories explain why uncertainty fluctuates using nonlinearities in a production economy (Van Nieuwerburgh and Veldkamp (2006), Fajgelbaum, Schaal, and Taschereau-Dumouchel (2013)) or active experimentation (Bachmann and Moscarini (2012)). Our model differs because it does not depend on an economic environment, only

\footnotetext{
${ }^{1}$ In contrast, Bachmann and Bayer (2012b) argue that there is little impact of uncertainty on economic activity.
} 
on a statistical law of motion. Our uncertainty shocks arise from agents applying Bayes law when parameters are unknown.

Our exercise also speaks to a set of papers that measure uncertainty shocks in various ways. Bloom (2009), Baker, Bloom, and Davis (2012), Stock and Watson (2012), Jurado, Ludvigson, and Ng (2013) Fernandez-Villaverde, Kuester, Guerron, and Rubio-Ramirez (2012), Fernandez-Villaverde, Guerron-Quintana, Rubio-Ramirez, and Uribe (2011), Nakamura, Sergeyev, and Steinsson (2012), Justiniano and Primiceri (2008) and Born, Peter, and Pfeifer (2011) document the properties of uncertainty shocks in the U.S. and in emerging economies, while Bachmann, Elstner, and Sims (2012) use forecaster data to measure ex-ante and ex-post uncertainty in Germany. While our paper also engages in a measurement exercise, we primarily contribute a quantitative model of how and why such shocks arise.

The idea that the probability of rare events is time-varying also appears in models of discrete, rare events (e.g., Rietz (1998), Barro (2006), Wachter (2013)). However, these papers assume that there is a time-varying probability of a rare event and derive the consequences of that for asset prices. Our paper is a complement to this literature; it describes a learning process that can generate volatile, counter-cyclical beliefs about the probabilities of extreme, negative outcomes. Using only the assumptions of Bayesian updating and the idea of parameter uncertainty in non-normal probability distributions, we show as a result that estimated probabilities of tail outcomes are volatile and that economic uncertainty is sensitive to these probabilities. This finding helps to justify the assumptions of the previous literature.

The theoretical part of our paper grows out of an existing literature that estimates Bayesian forecasting models with model uncertainty. Cogley and Sargent (2005) use such a model to understand the behavior of monetary policy, while Johannes, Lochstoer, and Mou (2011) estimate a similar type of model on consumption data to capture properties of asset prices. While the mechanics of model estimation are similar, the focus on skewed uncertainty shocks and uncertainty proxies distinguish our paper. Nimark (2012) also generates increases in uncertainty by assuming that only outlier events are reported. Thus, the publication of a signal conveys both the signal content and information that the true event is far away from the mean. Such signals can increase agents' uncertainty. But that paper 
does not attempt to quantitatively explain the fluctuations in uncertainty measures. Our paper is in the spirit of Hansen's Ely Lecture (Hansen, 2007) and Chen, Dou, and Kogan (2013) which advocate putting agents in the model on equal footing with an econometrician who is learning about his environment over time and struggling with model selection.

\section{A Linear-Normal Forecasting Model}

The purpose of the model is to explain why relaxing rational expectations and assuming that agents do not know the true distribution of outcomes with certainty opens up an additional source of uncertainty shocks. To isolate this new source of uncertainty shocks, we consider first a homoskedastic model. If an agent knew the true model (they had rational expectations), their uncertainty would be only about the random realizations of future shocks. Since the variance of these shocks is assumed to be constant over time, there would be no uncertainty shocks.

We consider a forecaster who observes real-time GDP growth data, in every quarter and forecasts the next period's growth. The agent knows that GDP growth comes from a linear model with a hidden state, but does not know the parameters of this model. Each period, he starts with prior beliefs about these parameters and the current state, observes the new GDP data, and updates his beliefs using Bayes' law.

\subsection{Definitions}

A model, denoted $\mathcal{M}$, has a vector a parameters $\theta$. Together, $\mathcal{M}$ and $\theta$ determine a probability distribution over a sequence of outcomes $y_{t}$. Let $y^{t} \equiv\left\{y_{\tau}\right\}_{\tau=0}^{t}$ denote a series of data available to the forecaster at time $t$. In every model, agent $i$ 's information set $\mathcal{I}_{i t}$ will include the model $\mathcal{M}$ and the history $y^{t}$ of $y$ observations up to and including time $t$. The state $S_{t}$, innovations, and the parameters $\theta$ are never observed.

The agent, who we call a forecaster and index by $i$, is not faced with any economic choices. He simply uses Bayes' law to forecast future $y$ outcomes. Specifically, at each date $t$, the agent conditions on his information set $\mathcal{I}_{i t}$ and forms beliefs about $y_{t+1}$. We call the expected value $E\left(y_{t+1} \mid \mathcal{I}_{i t}\right)$ an agent $i$ 's forecast and the square root of the conditional variance $\operatorname{Var}\left(y_{t+1} \mid \mathcal{I}_{i t}\right)$ is what we call uncertainty. Forecasters' forecasts will differ from 
the realized growth rate. This difference is what we call a forecast error.

Definition 1. An agent i's forecast error is the distance, in absolute value, between the forecast and the realized growth rate: $F E_{i, t+1}=\left|y_{t+1}-E\left[y_{t+1} \mid \mathcal{I}_{i t}\right]\right|$.

We date the forecast error $t+1$ because it depends on a variable $y_{t+1}$ that is not observed at time $t$. Similarly, if there are $N_{t}$ forecasters at date $t$, an average forecast error is

$$
\overline{F E} E_{t+1}=\frac{1}{N_{t}} \sum_{i=1}^{N_{t}} F E_{i, t+1} .
$$

We define forecast errors and uncertainty over 1-period-ahead forecasts because that is the horizon we focus on in this paper. But future work could use these same tools to measure uncertainty at any horizon.

Definition 2. Uncertainty is the standard deviation of the time- $(t+1)$ GDP growth, conditional on an agent's time-t information: $U_{i t}=\sqrt{E\left[\left(y_{t+1}-E\left[y_{t+1} \mid \mathcal{I}_{i t}\right]\right)^{2} \mid \mathcal{I}_{i t}\right]}$.

Volatility is the same standard deviation as before, but now conditional on the history $y^{t}$, the model $\mathcal{M}$ and the parameters $\theta$ :

Definition 3. Volatility is the standard deviation of the unexpected innovations in $y_{t+1}$, taking the model and its parameters as given:

$V O L_{t}=\sqrt{E\left[\left(y_{t+1}-E\left[y_{t+1} \mid y^{t}, \theta, \mathcal{M}\right]\right)^{2} \mid y^{t}, \mathcal{M}, \theta\right]}$.

If an agent knew the parameters (i.e., if $\mathcal{I}_{i t}=\left\{y^{t}, \mathcal{M}, \theta\right\}$ ), then uncertainty and volatility would be identical. Thus, the only source of uncertainty shocks would be volatility shocks.

Many papers equate volatility, uncertainty and squared forecast errors. These definitions allow us to understand the conditions under which these are equivalent. Volatility and uncertainty are both ex-ante measures because they are time- $t$ expectations of $t+1$ outcomes, which are time- $t$ measurable. However, forecast errors are an ex-post measure because it is not measurable at the time when the forecast is made. Substituting definition 1 into definition 3 reveals that $U_{i t}=\sqrt{E\left[F E_{i, t+1}^{2} \mid \mathcal{I}_{i t}\right]}$. So, uncertainty squared is the same as the expected squared forecast error. Of course, what people measure with forecast errors is typically not the expected squared forecast error. It is an average of realized squared forecast errors: $\sqrt{1 / N_{t} \sum_{i} F E_{i, t+1}^{2}}$. 


\subsection{The linear forecasting model}

We begin by examining the following continuous hidden-state process for $y_{t}{ }^{2}$

$$
\begin{aligned}
& y_{t}=\alpha+s_{t}+\sigma \varepsilon_{y, t} \\
& s_{t}=\rho s_{t-1}+\sigma_{s} \varepsilon_{s, t}
\end{aligned}
$$

where $\varepsilon_{y, t}$ and $\varepsilon_{s, t}$ are standard normal random variables independent of each other. In this model, the parameters are $\theta \equiv\left[\alpha, \rho, \sigma, \sigma_{s}\right]^{\prime}$.

Information assumptions Each forecaster has an identical information set: $\mathcal{I}_{i t}=$ $\left\{y^{t}, \mathcal{M}\right\}, \forall i$. The model $\mathcal{M}$ is described by (2) and (3). The state $s_{t}$ and the parameters $\theta$ are never observed.

To compute forecasts and the process for uncertainty, we use Bayesian updating A forecast is a conditional expectation of next-period growth, where the expectation is taken over unknown parameters, states, and growth realizations. Using the law of iterated expectations, we can write this forecast as:

$$
E\left(y_{t+1} \mid y^{t}\right)=\iiint y_{t+1} p\left(y_{t+1} \mid \theta, s_{t+1}, y^{t}\right) p\left(s_{t+1} \mid \theta, y^{t}\right) p\left(\theta \mid y^{t}\right) d \theta d s_{t+1} d y_{t+1}
$$

The first probability density function, $p\left(y_{t+1} \mid \theta, s_{t+1}, y^{t}\right)$, is the probability of $t+1$ GDP growth, given the state and the parameters. From 2, we know that, conditional on the state, GDP growth is normally distributed with mean $\alpha+s_{t+1}$ and variance $\sigma^{2}$.

The second probability density function, $p\left(s_{t+1} \mid \theta, y^{t}\right)$, is the probability of a hidden state in a Kalman filtering system. It is also conditionally normally distributed. When the parameters are known, (2) and (3) form the observation and state equations of a Kalman filtering system. The following recursive equations describe the conditional mean and variance of the first two probability terms, jointly:

$$
E\left[y_{t+1} \mid y^{t}, \theta, \mathcal{M}\right]=\rho E\left[y_{t} \mid y^{t-1}, \theta, \mathcal{M}\right]+\rho K_{t} y_{t}
$$

where the term $K_{t}=K_{t}=\left(I+\sigma^{2} \operatorname{Var}\left[y_{t} \mid y^{t-1}, \theta, \mathcal{M}\right]\right)^{-1}$ is the Kalman gain and the

\footnotetext{
${ }^{2}$ We have also explored a version of the model with a discrete hidden state. The results are very similar and are reported in Appendix B.
} 
conditional variance of the estimate is

$$
\operatorname{Var}\left[y_{t+1} \mid y^{t}, \theta, \mathcal{M}\right]=\left(\left(1-\rho^{2}\right) \sigma^{2}+\sigma_{S}^{2}\right) I
$$

Volatility is $\sqrt{\operatorname{Var}\left[y_{t+1} \mid y^{t}, \theta, \mathcal{M}\right]}$, which is a constant. Constant volatility may or may not be a realistic feature of the data. But it is a helpful starting point because it will allow us to isolate the fluctuations in uncertainty that come from parameter uncertainty.

Finally, the third probability density function is the probability of the parameter vector $\theta$, conditional on the $t$-history of observed GDP data. To compute posterior beliefs about parameters, we employ a Markov Chain Monte Carlo (MCMC) technique. ${ }^{3}$ At each date $t$, the MCMC algorithm produces a sample of possible parameter vectors, $\left\{\theta^{d}\right\}_{d=1}^{D}$, such that the probability of any parameter vector $\theta^{d}$ being in the sample is equal to the posterior probability of those parameters, $p\left(\theta^{d} \mid y^{t}\right)$. Therefore, we can compute an approximation to any integral by averaging over sample draws: $\int f(\theta) p\left(\theta \mid y^{t}\right) d \theta \approx 1 / D \sum_{d} f\left(\theta^{d}\right)$.

To estimate uncertainty, we compute these probability density terms and integrate numerically to get a forecast. In similar fashion, we also calculate $E\left(y_{t+1}^{2} \mid y^{t}\right)$. Applying the variance formula $\operatorname{Var}\left(y_{t+1} \mid y^{t}\right)=E\left(y_{t+1}^{2} \mid y^{t}\right)-E\left(y_{t+1} \mid y^{t}\right)^{2}$, and taking the square root yields uncertainty: $U_{t}=\sqrt{\operatorname{Var}\left(y_{t+1} \mid y^{t}\right)}$.

\subsection{Data Description}

There are two pieces of data that we use to evaluate and estimate our forecasting model. The first is real-time GDP data from the Philadelphia Federal Reserve. The variable we denote $y_{t}$ is the growth rate of GDP. Specifically, it is the log-difference of the real GDP series, times 400 , so that it can be interpreted as an annualized percentage change. We use real-time data because we want to accurately assess what agents know at each date. Allowing them to observe final GDP estimates, that are not known until much later, is not consistent with the goal. Therefore, $y_{t}$ represents the estimate of GDP growth between the end of quarter $t-1$ and quarter $t$, based on the GDP estimates available at time $t$. Similarly, $y^{t}$ is the history of GDP growth up to and including period $t$, based on the data available at time $t$.

\footnotetext{
${ }^{3}$ More details are presented in the Appendix.
} 
We use the second set of data, professional GDP forecasts, to evaluate our forecasting models. We describe below the four key moments that we use to make that assessment. The data come from the Survey of Professional Forecasters, released by the Philadelphia Federal Reserve. The data are a panel of individual forecaster predictions of real US output for both the current quarter and for one quarter ahead from quarterly surveys from 1968 Q4 to 2013 Q4. In each quarter, the number of forecasters varies from quarter-to-quarter, with an average of 40.5 forecasts per quarter.

Formally, $t \in\{1,2, \ldots, T\}$ is the quarter in which the survey of professional forecasters is given. Let $i \in\{1,2, \ldots, I\}$ index a forecaster and $I_{t} \subset\{1,2, \ldots, I\}$ be the subset of forecasters who participate in a given quarter. Thus, the number of forecasts made at time $t$ is $N_{t}=\sum_{i=1}^{I} \mathbb{I}\left(i \in I_{t}\right)$. Finally, let $y_{t+1}$ denote the GDP growth rate over the course of period $t$. Thus, if $G D P_{t}$ is the GDP at the end of period $t$, observed at the start of quarter $t+1$, then $y_{t+1} \equiv \ln \left(G D P_{t}\right)-\ln \left(G D P_{t-1}\right)$. This timing convention may appear odd. But we date the growth $t+1$ because it is not known until the start of date $t+1$.

\subsection{Estimation and results: linear-normal model}

Our forecaster needs prior distributions over all the parameters to start the updating process. We start with a flat prior, estimate each parameter on GDP growth data from 1947:2-1968:3, and use the mean and variance of this estimate as the mean and variance of prior beliefs. (See appendix for more details and prior estimation results.) Starting in quarter 4 of 1968, each period, the agent observes $y_{t}$ and revisions of previous quarters' data and updates his beliefs about future GDP growth using (4). We start the estimation of the model in 1968:4 because this is the first quarter for which we have forecasts from the Survey of Professional Forecasters.

To understand what properties of our forecasts come from parameter uncertainty, Figure 1 compares our uncertainty to that of an agent who takes the model parameters as given (labeled $\theta$ known). To keep the results comparable, the parameters that are used to compute volatility are the same as the mean prior beliefs in our model.

The main takeaway is that parameter uncertainty generates uncertainty shocks, albeit small ones. With known parameters, $\operatorname{stdev}\left(U_{t}\right)=0$. When parameters are updated every period, $\operatorname{stdev}\left(U_{t}\right)=0.48$. What triggers these uncertainty shocks is primarily surprises. 


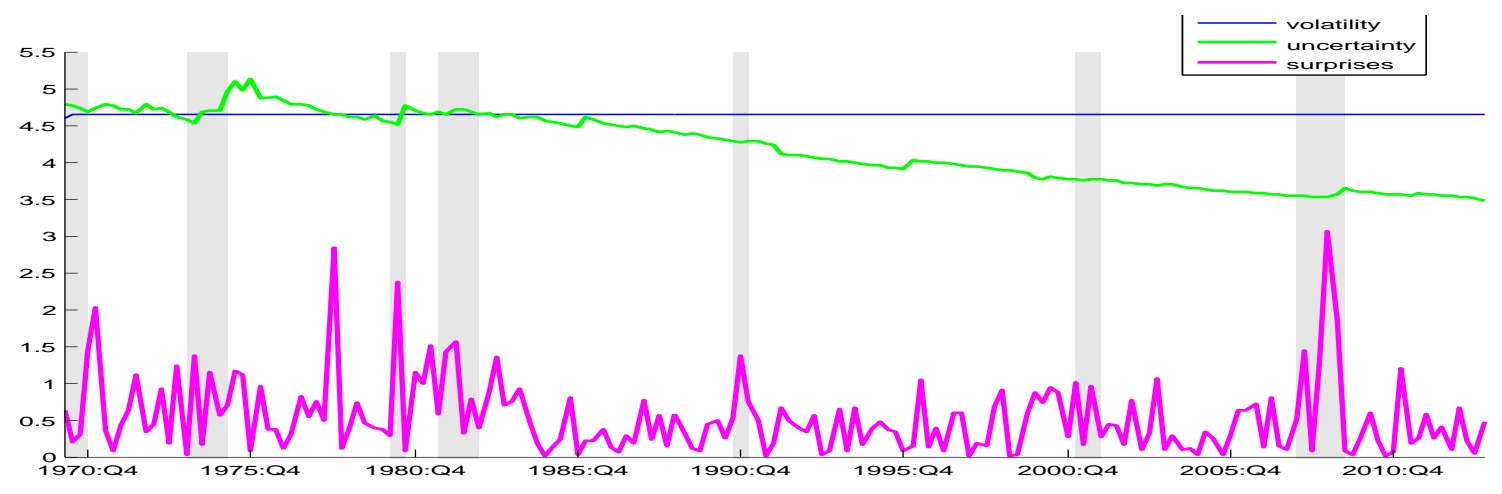

Figure 1: Uncertainty shocks in the linear model. Volatility, uncertainty and surprises are calculated using (6), definition 2 and (7).

We define a surprise to be an event that was unlikely, given the previous period's estimated model:

$$
\text { Surprise }_{t}=\frac{\left|y_{t}-E\left(y_{t} \mid y^{t-1}\right)\right|}{U_{t-1}} .
$$

Figure 1 plots this surprise measure against the uncertainty measure. What it reveals is that periods with large surprises are periods when uncertainty increases. But also that the uncertainty generated by the surprise persists, long after the surprise has passed.

Our results also expose three aspects of our forecasts that do not look realistic. 1) Our forecasters' uncertainty is not counter-cyclical $\left(\operatorname{Correl}\left(U_{t}, \mathrm{GDP}\right)=13 \%\right)$. Every common proxy for uncertainty is counter-cyclical and most theories use uncertainty to explain the onset of a recession. So, a forecasting model that fails to deliver this feature is suspect. 2) The model does not explain the low average forecasts of GDP observed in the professional forecaster data. The true average of GDP growth over 1968:Q4-2012:Q4 is 2.68\%. The average professional forecast of GDP growth is $2.24 \%$, almost half a percentage point lower. This model fails to explain that gap. ${ }^{4}$ 3) There is obviously no dispersion in forecasts. There is only one forecaster. Yet, forecast dispersion is a prominent and interesting feature of the data. Later, we will introduce heterogeneous signals to examine its role.

\footnotetext{
${ }^{4}$ This gap only arises in final GDP estimates. The average initial GDP announcement has $2.3 \%$ growth on average, in line with the forecasts. But if these initial announcements are themselves BEA forecasts of what the final GDP estimate will be, there is still a puzzle about why early estimates are systematically lower than final estimates.
} 
In the next section, we examine a nonlinear forecasting model that remedies the first two problems: It generates counter-cyclical uncertainty, forecasts that are low on average, and larger uncertainty shocks.

\section{A Skewed Forecasting Model}

The linear-normal model of the previous section was a starting point. It is a commonly-used framework where we shut down all sources of uncertainty shocks besides our estimation uncertainty and could see that mechanism at work. But that model misses important features of the forecast data and its uncertainty shocks are modest. One reason that uncertainty varies so little is that all the random variables are normally distributed. The normal distribution has the unusual properties that the conditional variance is the same, no matter what the conditional mean is and that an n-standard deviation event is always equally unlikely. Since uncertainty is a conditional variance, the normal distribution shuts down much scope for changes in uncertainty. Therefore, we explore a model that has non-normal shocks.

Moving away from normality, there is an infinite set of possibilities. But one natural choice is to focus on a distribution with skewness. Negative skewness is a salient feature of GDP data. In the real GDP (1968:Q4-2013:Q4) data we use for our forecasting model simulations, the skewness of GDP growth is -0.30 . Skewness is also a feature of many models. Models where workers lose jobs quickly and find jobs gradually, or models where borrowing constraints amplify downturns are just a couple of examples of models that generate booms that are more gradual than crashes. Finally, we find that a model with skewness both does a better job of matching features of forecast data and generates much larger uncertainty shocks.

Updating non-normal variables is typically cumbersome. Combining this with parameter uncertainty typically requires particle filtering, which is possible, but slow. We make this problem tractable by doing a change of measure. The Radon-Nikodym theorem tells us that, for any measure $g$ that is absolutely continuous with respect to a measure induced by a normal distribution, we can find a change-of-measure function $f$ such that $g(x)=\int f(x) d \Phi(x)$, where $\Phi$ is a normal cdf. If we estimate such an $f$ function, then we use $f^{-1}$ to take data from a skewed distribution and transform it into normal data that 
we can then use the tools from the last section to learn about.

Of course, allowing a forecaster to explore the whole function space of possible $f$ 's is not viable. We focus the problem by considering a family of functions and allowing the forecaster to consider parameter estimates that govern the properties of the distribution. The change of measure function should have three desirable properties: 1) Its range is the real line; 2) it is monotone; and 3) it can be either globally concave or globally convex, depending on the estimated parameters. A class of transformations that satisfy this criteria is ${ }^{5}$

$$
f\left(\widetilde{X}_{t}\right)=c+b \exp \left(-\widetilde{X}_{t}\right)
$$

If we have estimates for $b$ and $c$, we can do a change of variable: Use $f^{-1}\left(y_{t}\right)$ to transform GDP growth into a variable $\widetilde{X}$, which is a normally-distributed continuous variable with a hidden persistent state.

$$
\begin{aligned}
\tilde{X}_{t} & =S_{t}+\sigma \epsilon_{t} \\
S_{t} & =\rho S_{t-1}+\sigma_{S} \varepsilon_{t}
\end{aligned}
$$

where $\epsilon_{t} \sim N(0,1)$ and $\varepsilon_{t} \sim N(0,1)$. This procedure allows our forecaster to consider a family of non-normal distributions of GDP growth and convert each one into a linearnormal filtering problem with unknown parameters that can be solved using the same tools as in the previous section. The only additional complication is that the parameters $b$ and $c$ also need to be estimated.

The $b$ parameter governs the mean of the $\widetilde{X}$ process. To see this, note that for $b<0$, we can rewrite $b \exp \left(-\widetilde{X}_{t}\right)=-\exp \left(-\widetilde{X}_{t}+\ln (|b|)\right)$. To streamline our code, we simply remove the time- $t$ sample mean of the $\widetilde{X}_{t}$ and set $b=-1$. After estimating the parameters of the mean-zero process, we add back in the sample mean. This approach is supported by the fact that when we have estimated $b$ in less complex settings, we come up with consistently negative values and quantitatively similar estimates.

We start with priors (see Appendix) and use MCMC techniques to form beliefs about

\footnotetext{
${ }^{5}$ Of course, the choice of class of transformations can affect our uncertainty estimates. However, the key property of this function is its degree of curvature. We allow our agent to estimate a parameter that governs this curvature. Any function with similar curvature, such as a polynomial or sine function, would have a similar mechanism. Our work in progress is exploring a concave, piecewise polynomial function.
} 
the $\rho, \sigma, \sigma_{s}$ and $c$ parameters. For each parameter draw $\theta_{i}$ from the MCMC algorithm, we compute $E\left[y_{t} \mid \mathcal{I}_{t}, \theta_{i}\right]$ and $E\left[y_{t}^{2} \mid \mathcal{I}_{t}, \theta_{i}\right]$. We average these expectations over all parameter draws and compute uncertainty as $U_{t}=E\left[y_{t}^{2} \mid \mathcal{I}_{t}\right]-E\left[y_{t} \mid \mathcal{I}_{t}\right]^{2}$.

\subsection{Results: Uncertainty Shocks}

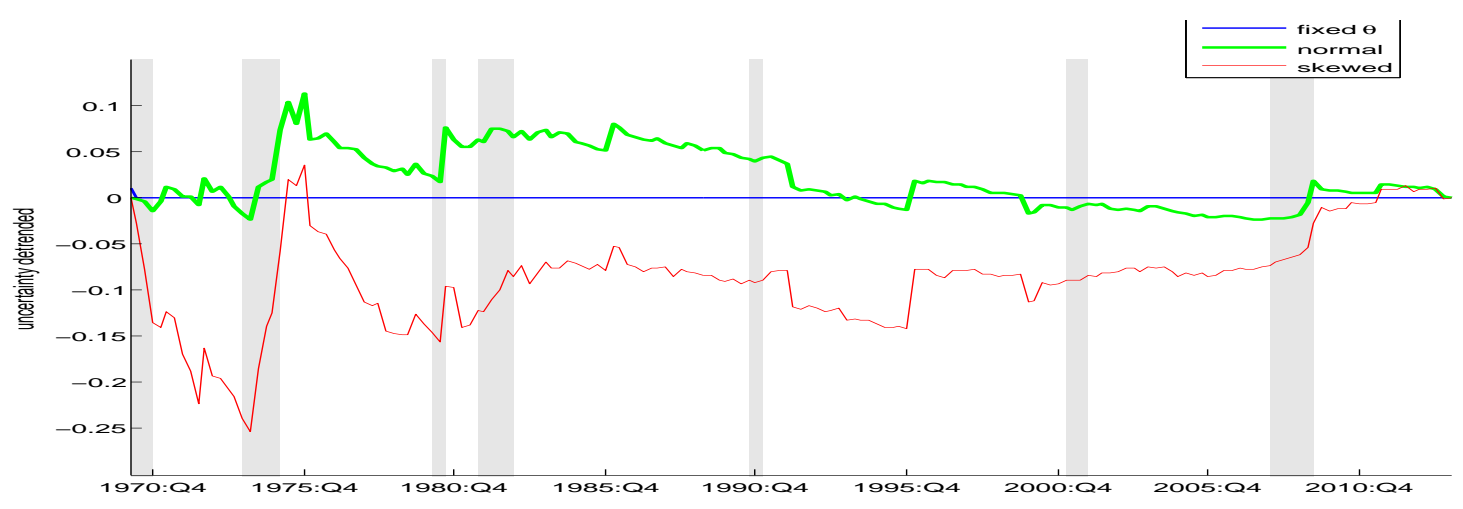

Figure 2: Uncertainty $\left(U_{t}\right)$ implied by linear and skewed models in log deviations from trend.

Figure 2 compares the time series of uncertainty in both models. Column (3) of Table 1 shows that updating beliefs about the skewness of the GDP growth distribution has a large effect on uncertainty. Such learning increases the average level of uncertainty by only $8 \%$. But it amplifies uncertainty shocks. The standard deviation of the uncertainty series was $0.48 \%$ with normally-distributed outcomes and rises to $1.50 \%$ when our forecaster updates beliefs about skewness. One can interpret the magnitude of this standard deviation relative to the mean. A 1-standard deviation shock to uncertainty raises uncertainty $33 \%$ above its mean. That is quite a volatile process and offers a stark contrast to the relatively modest changes in volatility typically measured.

Since using growth rates of GDP is a form of trend-removal, it makes sense to correlate a stationary series with another stationary series. Therefore, we detrend volatility and uncertainty in order to discern the nature of their cyclical components (Table 1, middle panel). We remove the trend in uncertainty using log deviations from an exponential trend:

$$
\tilde{U}_{t} \equiv \ln \left(U_{t}\right)-\ln \left(U_{t}^{\text {trend }}\right)
$$




\begin{tabular}{|c|c|c|c|}
\hline model: & & normal & skewed \\
\hline \multirow[t]{2}{*}{ Mean } & $\overline{U_{t}}$ & $4.20 \%$ & $4.53 \%$ \\
\hline & $V_{t}$ & $4.65 \%$ & $5.41 \%$ \\
\hline \multirow[t]{2}{*}{ Std deviation } & $U_{t}$ & $0.48 \%$ & $1.50 \%$ \\
\hline & $V_{t}$ & $0 \%$ & $0.07 \%$ \\
\hline \multirow[t]{3}{*}{ Autocorrelation } & $U_{t}$ & 0.99 & 0.97 \\
\hline & $V_{t}$ & 0 & 0.92 \\
\hline & \multicolumn{3}{|c|}{ Detrended uncertainty/volatility } \\
\hline $\operatorname{Corr}\left(\tilde{U}_{t}, E_{t}\left[y_{t+1}\right]\right)$ & & 0.04 & -0.78 \\
\hline \multirow[t]{3}{*}{$\operatorname{Corr}\left(\tilde{V}_{t}, E_{t}\left[y_{t+1}\right]\right)$} & & 0 & -0.99 \\
\hline & \multicolumn{3}{|c|}{ Forecast properties } \\
\hline & data & normal & skewed \\
\hline Mean forecast & $2.29 \%$ & $2.82 \%$ & $2.27 \%$ \\
\hline Mean $|F E r r|$ & $1.87 \%$ & $2.25 \%$ & $2.51 \%$ \\
\hline Std forecast & $2.25 \%$ & $1.17 \%$ & $0.64 \%$ \\
\hline Std $|F E r r|$ & $1.46 \%$ & $2.17 \%$ & $2.39 \%$ \\
\hline
\end{tabular}

Table 1: Properties of model uncertainty series. Forecasts are computed using equation (4). Forecast error is (forecast - final GDP growth). Volatilities are computed assuming that the true parameters $\theta$ are equal to the the mean prior beliefs for the parameter learning models.

The resulting series, plotted in figure 2, reveals large, highly counter-cyclical uncertainty shocks. Not only is the level higher, uncertainty rose noticeably during each of the recessions since 1970 .

Keep in mind that there is still no stochastic volatility in this model. To the extent that we believe that there are volatility shocks to GDP, this would create additional shocks to uncertainty, above and beyond those we have already measured. These series are not yet a complete picture of macroeconomic uncertainty. Instead, they are a look at what part of uncertainty is missed when we just measure volatility.

Clearly, the model is not forecasting GDP as accurately as the forecasters in the survey of professional forecasters do (Table 1, bottom panel). However, this is a problem that we can remedy, without changing our main message. The forecasts in the model are based only on prior GDP releases. In reality, forecasters have access to other sources of data that improve the accuracy of their forecasts. The fact that the model produces a forecast error that is too large and too volatile reflects this problem. See section 3.2 for a sketch 
of a model with additional forecasting information that remedies this problem, without undermining our uncertainty shock mechanism.

Finally, uncertainty is very persistent. It is highest at the start of the sample, when data is most scarce, and then slowly decays over the rest of the sample. As noted by CollinDufresne, Johannes, and Lochstoer (2013), the persistent uncertainty process comes from the nature of learning: A single large shock to GDP growth results in a quick reevaluation of the parameter and model probabilities. These revisions in beliefs act as permanent, non-stationary shocks even when the underlying shock is transitory.

\subsection{Skewness Shocks and Time-Varying Black Swan Risk}

To understand why uncertainty varies so much, it is helpful to look at the probability of tail events. Since our estimated probability distribution is negatively skewed, negative outliers are more likely than positive ones. For a concrete example, let's consider the probability of a particular negative growth event. The mean of GDP growth is $2.68 \%$, while its standard deviation is $3.32 \%$. If GDP growth were normally distributed, then $y_{t+1} \leq-6.8 \%$ would be a 1-in-100-year event $(\operatorname{Pr}=0.0025$ quarterly $)$. Let's call this rare event a black swan.

$$
\text { Black Swan Risk } k_{t}=\operatorname{Prob}\left[y_{t+1} \leq-6.8 \% \mid \mathcal{I}_{t}\right]
$$

The correlation between black swan risk and uncertainty is $97 \%$ ( $75 \%$ for the detrended series). This illustrates that uncertainty shocks arise in times when the estimated probabilities of extreme events change. Our model suggests that uncertainty builds up gradually over time as more and more unusual observations are realized.

When the distribution of GDP growth is non-normal and states and parameter estimates change over time, the probability of this black swan event fluctuates. Figure 3 plots the estimated black swan probability each period. The black swan probability varies considerably. It reaches a high of $18 \%$ in early 1970. Leading up to the 2008 financial crisis, the black swan probability rose from 3.5\% in 2007 Q1 to over 4.6\% in Q3 of 2009.

These results teach us that when we include parameter uncertainty in our notion of economic uncertainty, and we consider a model with skewed outcomes, then most changes in uncertainty coincide with changes in the estimated probability of rare events. Most of 


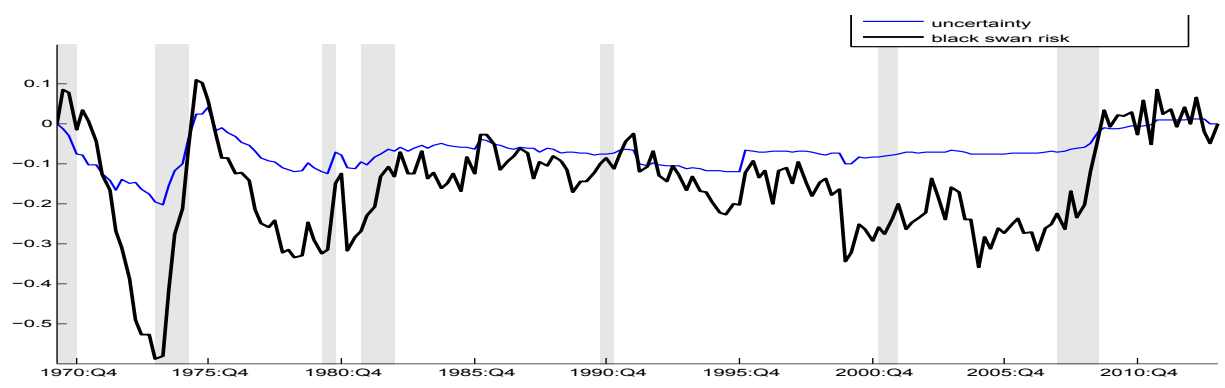

Figure 3: When the probability of a black swan event is high, uncertainty is high. Black Swan Risk is defined in (10). It is evaluated in the model where the skewness parameter $c$ is updated every period.

these uncertainty shocks were not present when we did not allow the forecaster to update his skewness beliefs. When we allow for learning about skewness, new pieces of data cause changes in the skewness estimates. Tail event probabilities are very sensitive to this skewness parameter. When the probability of extreme events is high, uncertainty is high as well.

This explanation raises the question: What types of data realizations make estimated skewness more negative, increase black swan risk, and thereby generate uncertainty shocks? We find two types of episodes that set up large uncertainty shocks. The first is simply a large negative GDP growth realization. When a negative outlier is observed, the forecaster revises skewness to be more negative and increases the estimated variance of shocks, both of which cause the probability of a black swan event and uncertainty to rise. This is what happens in 2008 and in the early 1980's. But there is a second, more subtle, cause of uncertainty shocks that comes from a sequence of mild positive GDP growth realizations in a row. These observations cause the forecaster to increase the estimated mean of the distribution. When the mean increases, the existing negative outlier data points become further from the mean. Because the previously-observed negative realizations are more extreme, the estimate of skewness rises and the probability of rare negative events can rise as well. This is what happens in the early 1970's. A sequence of positive growth realizations causes a rise and then a fall in uncertainty. But the persistence of the high estimated skewness set the stage for the large rise in uncertainty in the second half of the 1970's. This mechanism provides one explanation for why uncertainty seems to rise 
particularly at the end of long spells of consistently positive growth.
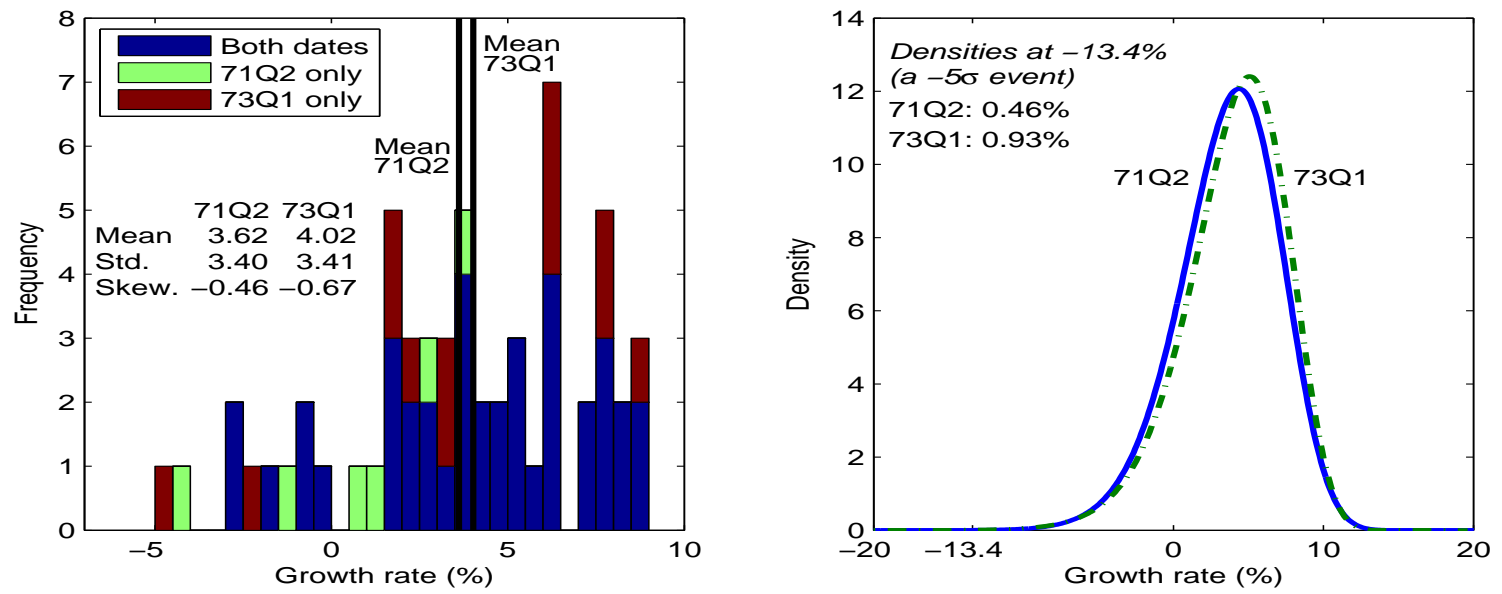

Figure 4: An example of a positive growth episode that increased the estimated mean, skewness and black swan probability.

\subsection{Negative Skewness as a Force for Counter-Cyclical Uncertainty}

One way of understanding the cyclical effect skewness has on uncertainty is by thinking about the skewed distribution as a non-linear transformation of a normal variable. The non-linear transformation has no economic interpretation. It does not represent a utility function, production function or anything other than an estimated change-of-measure function that regulates the skewness of outcomes. ${ }^{6}$ But since many problems in economics use normal shocks and compute means and variances of concave functions of these shocks, we can leverage that intuition here to understand the role of skewness. (See Albagli, Hellwig, and Tsyvinski (2013) for a similar approach.)

The unconditional distribution of GDP growth rates is negatively skewed. Therefore, when we estimate the change of measure function $f(8)$, we consistently find that the

\footnotetext{
${ }^{6}$ Although this paper does not try to explain the negative skewness of outcomes, many other theories do. Negative skewness can arise when the economy is functioning very well (high $\widetilde{X}_{t}$ ), then improving its efficiency results in a small increase in GDP. But if there is a high degree of dysfunction or inefficiency (low $\widetilde{X}$ ), then the economy can fall into depression. Many models generate exactly this type of effect through borrowing or collateral constraints, other financial accelerator mechanisms, matching frictions, or information frictions. Even a simple diminishing returns story could explain such skewness.
} 
coefficient $b$ is negative, meaning that the transformation is concave. A concave transformation of a normal variable puts more weight on very low realizations and makes very high realizations extremely unlikely. In other words, the concave transformation creates a negatively-skewed variable.

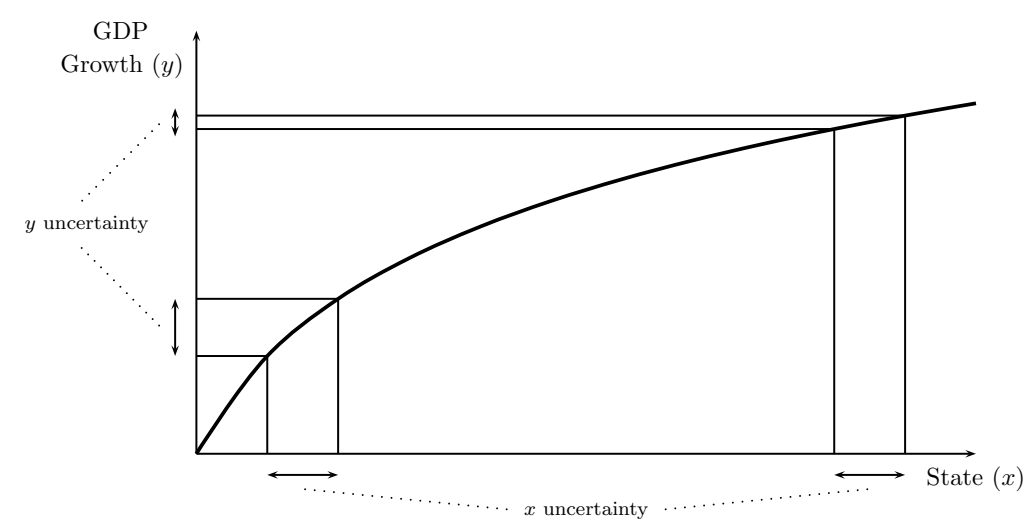

Figure 5: Nonlinear change of measure and counter-cyclical uncertainty. A given amount of uncertainty about $x$ creates more uncertainty about $y$ when $x$ is low than it does when $x$ is high.

The concavity of the change of measure explains why uncertainty becomes countercyclical. The concave line in Figure 5 is a mapping from $x$ into GDP growth, $y$. The slope of this curve is a Radon-Nikodym derivative. A given amount of uncertainty is like a band of possible $x$ 's. (If $x$ was uniform, the band would represent the positive-probability set and the width of the band would measure uncertainty about $x$.) If that band is projected on to the $y$-space, the implied amount of uncertainty about $y$ depends on the state $x$. When $x$ is high, the mapping is flat, and the resulting width of the band projected on the $y$-axis ( $y$ uncertainty) is small. When $x$ is low, the band projected on the $y$ axis is larger and uncertainty is high. (See Straub and Ulbricht (2013) for theoretical results relating concavity to uncertainty.)

Learning about skewness causes this concave curve to shift over time. When a negative outlier is observed, the estimated state falls and estimated skewness becomes more negative. More skewness translates into more curvature in the change of measure function. Combined with a low estimated state, this generates even more uncertainty. Thus, bad events trigger larger increases in uncertainty. This is reflected is the more negative correlation between forecasts and uncertainty in the learn skew model in Table 1. 


\subsection{Why Skewness and Model Uncertainty Lower Forecasts}

Aside from generating larger uncertainty shocks, the model with skewness also explains the low GDP growth forecasts in the professional forecaster data. The average forecast is $2.27 \%$ in the model and $2.29 \%$ in the forecaster (SPF) data. ${ }^{7}$ Thus, the first puzzle is why the nonlinear model produces these average forecasts. If GDP growth is a concave transformation of a linear-normal underlying variable, Jensen's inequality tells us that expected values will be systematically lower than the median realization. But by itself, Jensen's inequality does not explain the forecast bias because the expected GDP growth and the mean GDP growth should both be lowered by the concave transformation (see figure 6 , left panel). It must be that there is some additional uncertainty in expectations,
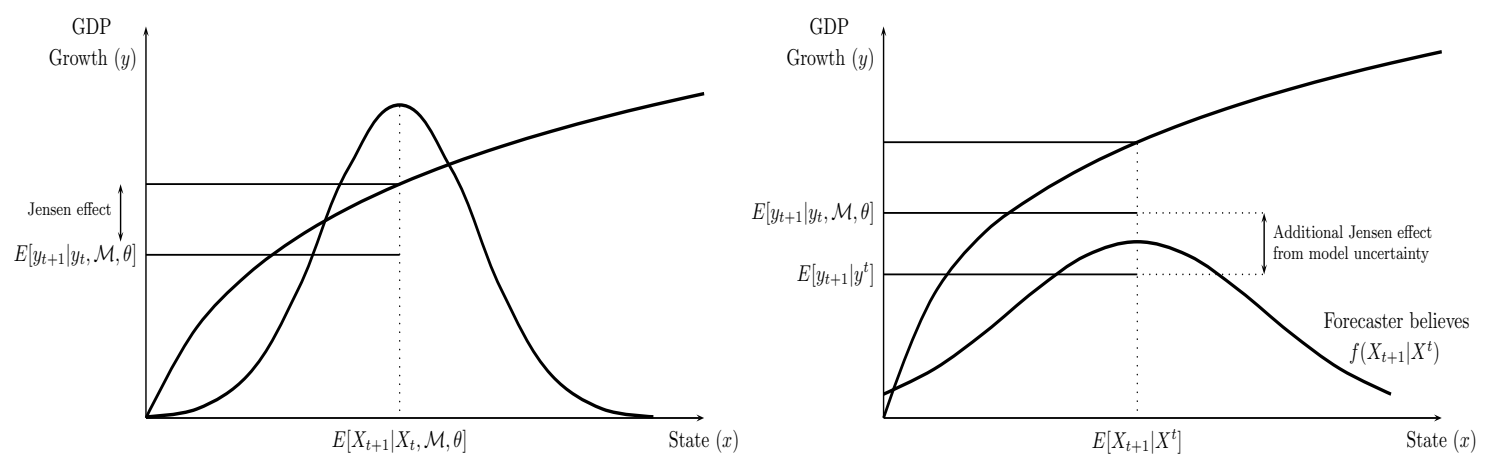

Figure 6: Explaining why average forecasts are lower than mean GDP growth. The result has two key ingredients: The forecaster faces more uncertainty than he would if he knew the true distribution of outcomes, and a Jensen inequality effect from the concave change of measure.

making the Jensen inequality effect larger for forecasts than it is for the unconditional mean of the true distribution (see figure 6, right panel). This would explain why our results tell us that most of the time the sample mean is greater than the average forecast. If the agent knew the true parameters, he would have less uncertainty about $y_{t+1}$. Less uncertainty would make the Jensen effect smaller and raise his estimate of $y_{t+1}$, on average. Thus, it is the combination of parameter uncertainty and a skewed distribution that can explain the forecast bias. ${ }^{8}$

\footnotetext{
${ }^{7}$ Elliott and Timmermann (2008) confirm our finding of forecast bias. They argue that stock analysts over-estimate earnings growth and the Federal Reserve under-estimates GDP growth.

${ }^{8}$ When we compare SPF forecasts to first-release GDP data, the difference in means disappears. But that is consistent with an interpretation of this first release as itself a forecast. The first release may be
} 


\subsection{Convergence and the Downward Trend in Uncertainty}

Since the true parameters in this model are constant, eventually agents will learn them, if the model is correctly specified. Even in our 44-year sample, there is evidence of convergence. There is a downward trend in uncertainty, some of which comes from the decline in the uncertainty about the parameter values. Between 1970 and 2013, uncertainty falls from $6.2 \%$ to $3.5 \%$. Does this decline imply that all parameter uncertainty should be resolved in the near future and these effects will disappear? There are three reasons why parameter uncertainty would persist.

First, our forecasting model is clearly not a complete or correct description of the macroeconomy. Our simple specification represents the idea that people use simple models to understand complex economic processes. Bayesian learning converges when the model is correctly specified. But when the estimated model and the true data-generating process differ, there is no guarantee that parameter beliefs will converge to the truth. Even as the data sample becomes large, parameter beliefs can continue to fluctuate, generating uncertainty shocks.

Second, much of the trend decline in uncertainty comes from lower estimated volatility. The mean estimate of the transitory shock variance $\left(\sigma^{2}\right)$ falls by $46 \%$ between 1970 Q1 and 2013 Q4. The mean estimate of variances decline because GDP growth just becomes less volatile in the second half of the sample and agents react to that by revising down their estimates of the variance parameters. Lower innovation variance also reduces uncertainty.

Finally, simply adding time-varying parameters can avoid convergence. If we assume that some or all of the parameters drift over time, then beliefs about these parameters will continue to change over time, just like beliefs about the hidden state $s_{t}$ fail to converge over time. One example of a model with time-varying parameters is a stochastic volatility model. Our estimation of a stochastic volatility model with learning about the volatility and the other fixed parameters of the model is still work in progress.

lower, on average because it is more uncertain and the added uncertainty, combined with the non-linear model, lowers the average initial announcement. 


\section{Data Used to Proxy for Uncertainty}

Our model generates a measure of economic uncertainty. Next, we compare our measure to others. Commonly used measures for uncertainty include the VIX, forecast dispersion, squared forecast errors, and GARCH volatility estimates. First, we describe each measure and examine the theoretical difference with ours. Then, we compare the statical properties.

\subsection{Time-varying volatility models}

One common procedure for estimating the size of volatility shocks is to estimate an ARMA process that allows for stochastic volatility. In order to compare such a volatility measure to our uncertainty series, we estimate a GARCH model of GDP growth, that allows for time-variation in the variance of the innovations. All data is quarterly and all of these series are non-stationary. To obtain stationary series, we use annualized growth rates.

The GARCH process that generates the best fit is one with an AR(1) process for GDP growth $(y)$ and a $\operatorname{GARCH}(1)$ process for volatility, $\sigma_{t+1}^{2}$, which includes 1 lagged variance:

$$
\begin{gathered}
y_{t+1}=3.38+0.41 y_{t}+\epsilon_{t+1}, \quad \epsilon_{t+1} \sim \mathcal{N}\left(0, \sigma_{t+1}^{2}\right) \\
\sigma_{t+1}^{2}=0.52+0.76 \sigma_{t}^{2}+0.24 \epsilon_{t}^{2}
\end{gathered}
$$

We assume that the errors in our models, $\epsilon_{t}$, are Gaussian and estimate the process using our full sample of data (1947-2012). The complete set of estimates, stationarity tests, as well as more detail about the model selection process are reported in appendix B.

Our forecasting model assumed that innovations to the state and observation equations had constant variance. We estimate homoskedastic models to test the hypothesis of homoskedasticity with an ARCH-LM test. The evidence for time-varying volatility is inconclusive. $^{9}$

\footnotetext{
${ }^{9}$ The log likelihood of the highest-likelihood heteroskedastic model is only $3 \%$ higher than the best-fitting homoskedastic model. With an ARCH-LM test, one cannot reject the null hypothesis of homoskedasticity (pvalue is 0.22 ). We come to a similar conclusion if we estimate the model, starting in 1947, use a stochastic volatility model, or relax the distributional assumptions. Our baseline analysis assumes that errors $\epsilon_{t}$ have a Gaussian distribution. Using distributions with fatter tails (student- $t$ ) yields no difference in estimations or significance. Furthermore, we explore further lags of all variables; either coefficients were not significantly different from zero or the log-likelihood was reduced. Finally, we included different lags of linear terms for $\epsilon_{t}$ and variances $\sigma_{t}^{2}$ in the GARCH specification. Again, the estimated parameters were not significant or
} 
But we could incorporate volatility shocks in our forecasting model by allowing the shock variances $\sigma^{2}$ and/or $\sigma_{s}^{2}$ to have their own stochastic processes. Increases in volatility would increase uncertainty directly and indirectly, by making other parameters harder to learn. Future work will explore this interaction.

\subsection{Forecast dispersion}

Some authors use forecast dispersion $\left(D_{t}\right.$ in equation 14) as a measure of uncertainty. ${ }^{10}$ One advantage of this measure is that it is typically regarded as "model-free." It turns out that dispersion is not model-free. It is only equivalent to uncertainty in models with uncorrelated signal noise and no parameter uncertainty.

Any unbiased forecast can be written as the difference between the true variable being forecast and some forecast noise that is orthogonal to the forecast. In other words,

$$
y_{t+1}=E\left[y_{t+1} \mid \mathcal{I}_{t}\right]+\eta_{t}+e_{i t}
$$

where the forecast error $\left(\eta_{t}+e_{i t}\right)$ is mean-zero and orthogonal to the forecast. We can further decompose any forecast error into a component that is common to all forecasters $\eta_{t}$ and a component that is the idiosyncratic error $e_{i t}$ of forecaster $i$.

Dispersion is the average squared difference of each forecast from the average forecast. We can write each forecast as $y_{t+1}-\eta_{t}-e_{i t}$. Then, with a large number of forecasters, we can apply the law of large numbers, set the average $e_{i t}$ to 0 and write the average forecast as $\bar{E}\left[y_{t+1} \mid \mathcal{I}_{t}\right]=y_{t+1}-\eta_{t}$. Thus,

$$
D_{t} \equiv \frac{1}{N} \sum_{i}\left(E\left[y_{t+1} \mid \mathcal{I}_{t}\right]-\bar{E}\left[y_{t+1} \mid \mathcal{I}_{t}\right]\right)^{2}=\frac{1}{N} \sum_{i} e_{i t}^{2}
$$

Whether dispersion accurately reflects uncertainty depends on private or public nature of information. Imprecise private information (high $e_{i t}^{2}$ ) generates forecast dispersion, while imprecise public information or estimation error typically does not.

To understand the importance of this distinction, Orlik, Veldkamp, and Kozeniauskas (2013) explore a forecasting model with forecast dispersion. In that model, signals are a the log-likelihood was reduced.

${ }^{10}$ See e.g. Baker, Bloom, and Davis (2012) or Diether, Malloy, and Scherbina (2002), or Johnson (2004). 
stand-in for all this other macroeconomic data that forecasters can use. Forecasters update beliefs as in section 1. But each period, each forecaster $i$ observes an additional signal $z_{i t}$ that is the next period's GDP growth, with common signal noise and idiosyncratic signal noise:

$$
z_{i t}=y_{t+1}+\eta_{t}+\epsilon_{i t}
$$

where $\eta_{t} \sim N\left(0, \sigma_{\eta}^{2}\right)$ is common to all forecasters and $\epsilon_{i t} \sim N\left(0, \sigma_{\epsilon}^{2}\right)$ is i.i.d. across fore-

casters. The two signal noise variances $\sigma_{\eta}^{2}$ and $\sigma_{\epsilon}^{2}$ match the average dispersion of forecasts and the average forecast error, $1 / T \sum_{t} F E_{t}$.

The main result is that forecast dispersion reduces uncertainty and average forecast errors, in line with the data. But it does not create uncertainty shocks. Parameters are estimated on a long string of historical data. That data is common to all forecasters. The differences in signals about next-quarter GDP growth create differences in forecasts. But for parameter estimation, there are many pieces of data that forecasters have in common and one signal that differs. So the parameter estimates mostly reflect the common historical data that forecasters observe. Since they have little heterogeneity, they do not contribute to changes in forecast dispersion. Estimation error looks more like the public component of the forecast error than it does the private component.

The bottom line is that forecast dispersion is not synonymous with uncertainty. Building a quantitative model with forecast dispersion is not a mechanism for generating large uncertainty shocks. Uncertainty shocks that come from parameter uncertainty are unlikely to be captured by a dispersion measure because most of the relevant data used for estimation is public, not private.

\subsection{Mean-squared forecast errors}

A measure related to forecast dispersion that captures both private and common forecast errors is the forecast mean-squared error.

We define a forecast mean-squared error $\left(M S E_{t+1}\right)$ of a forecast of $y_{t+1}$ made in quarter $t$ as the square root of the average squared distance between the forecast and the realized value

$$
M S E_{t+1}=\sqrt{\frac{\sum_{i \in I_{t}}\left(E\left[y_{t+1} \mid \mathcal{I}_{i t}\right]-y_{t+1}\right)^{2}}{N_{t}}} .
$$


If forecast errors were completely idiosyncratic, with no common component, then dispersion in forecasts and mean-squared forecasting errors would be equal. To see this, note that $F E_{j t}^{2}=\left(E\left[y_{t+1} \mid \mathcal{I}_{j t}\right]-y_{t+1}\right)^{2}$. We can split up $F E_{j t}^{2}$ into the sum $\left(\left(E\left[y_{t+1} \mid \mathcal{I}_{j t}\right]-\right.\right.$ $\left.\left.\bar{E}_{t}\left[y_{t+1}\right]\right)+\left(\bar{E}\left[y_{t+1}\right]-y_{t+1}\right)\right)^{2}$, where $\bar{E}_{t}\left[y_{t+1}\right]=\int_{j} E\left[y_{t+1} \mid \mathcal{I}_{j t}\right]$ is the average forecast. If the first term in parentheses is orthogonal to the second, $1 / N \sum_{j} F E_{j t}^{2}=M S E_{t}^{2}$ is simply the sum of forecast dispersion and the squared error in the average forecast: $E\left[y_{t+1} \mid \mathcal{I}_{j t}\right]-$ $\left.\bar{E}_{t}\left[y_{t+1}\right]\right)^{2}+\left(\bar{E}_{t}\left[y_{t+1}\right]-y_{t+1}\right)^{2}$.

We can then use this insight along with our forecast data to evaluate the extent to which variation in mean-squared errors (MSE) comes from changes in the accuracy of average forecasts and how much comes from changes in dispersion. We estimate a regression of the GDP forecast mean-squared error, $M S E^{2}$, defined in (16) on $\left(\bar{E}_{t}\left[y_{t+1}\right]-y_{t+1}\right)^{2}$. We find that the $R^{2}$ of this regression is $80 \%$. The remaining variation is due to changes in forecast dispersion. Since most of the fluctuation in MSE comes from changes in average forecast errors, it tells us that using forecast dispersion as a proxy for uncertainty will miss an important source of variation.

\subsection{VIX and confidence measures}

We discuss the volatility and forecast-based measures in most detail because there are measures that we can relate explicitly to our theory. Other proxy variables for uncertainty are interesting but have a less clear connection to our model. The market volatility index (VIX) is a traded blend of options that measures expected percentage changes of the S\&P500 in the next 30 days. It captures expected volatility of equity prices. But it would take a rich and complicated model to link macroeconomic uncertainty to precise movements in the VIX. Nevertheless, we can compare its statistical properties to those of the uncertainty measure in our model. Figure 7 does just this.

Another commonly cited measure of uncertainty is business or consumer confidence. The consumer confidence survey asks respondents whether their outlook on future business or employment conditions is "positive, negative or neutral." Likewise, the index of consumer sentiment asks respondents whether future business conditions and personal finances will be "better, worse or about the same." While these indices are indeed negatively correlated with the GARCH-implied volatility of GDP, they are not explicitly questions about uncertainty. 
Furthermore, we would like to use a measure that we can compare to the forecasts in our model. Since it is not clear what macro variable "business conditions" or "personal finances" corresponds to, it is not obvious what macro variable respondents are predicting.

\subsection{Comparing uncertainty proxies to model-generated uncertainty}

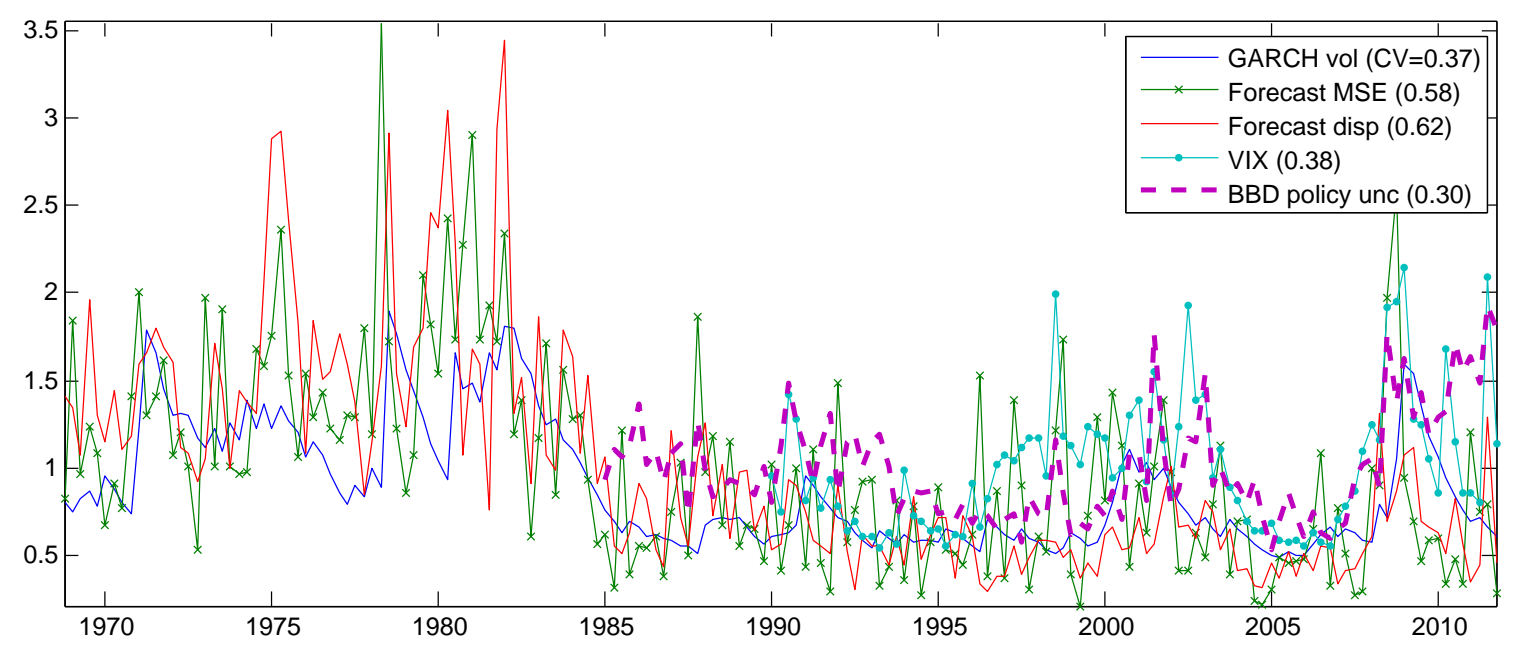

Figure 7: Comparing variables used to measure uncertainty in the literature.

Figure 7 plots each of the uncertainty proxies. There is considerable comovement, but also substantial variation in the dynamics of each process. These are clearly not measures of the same stochastic process, each with independent observation noise. Furthermore, they have properties that are quite different from our model-implied uncertainty metric. Table 2 shows that our uncertainty metric is negatively correlated with traditional measures of volatility, but is highly correlated with the volatility index (VIX) and the Baker, Bloom, and Davis (2012) policy uncertainty index.

\subsection{Inferring uncertainty from probability forecasts}

One way to infer the uncertainty of an economic forecaster is to ask them about the probabilities of various events. The survey of professional forecasters does just that. They ask about the probability that GDP growth exceeds $6 \%$, is between 5-5.9\%, between 4- 


\begin{tabular}{lccccl}
\hline \hline & Mean & $\begin{array}{l}\text { Standard } \\
\text { deviation }\end{array}$ & autocorr & $\begin{array}{c}\text { correlation } \\
\text { with } g_{t+1}\end{array}$ & $\begin{array}{l}\text { correlation } \\
\text { with } \tilde{U}_{t}\end{array}$ \\
\hline forecast MSE & $2.64 \%$ & 1.53 & 0.48 & 0.04 & $-20.8 \%$ \\
forecast dispersion & $1.54 \%$ & 0.95 & 0.74 & -0.19 & $-15.4 \%$ \\
GARCH volatility & $3.65 \%$ & 1.35 & 0.90 & 0.06 & $-22.3 \%$ \\
VIX & 20.55 & 7.81 & 0.58 & -0.41 & $40.2 \%$ \\
BBD policy uncertainty & 105.95 & 31.79 & 0.65 & -0.41 & $56.0 \%$ \\
\hline \hline
\end{tabular}

Table 2: Properties of forecast errors and volatility series for macro variables. Coefficient of variation (CV) listed in parentheses. Forecast MSE and dispersion are defined in (16) and (14) and use data from 1968q4-2011q4. Growth forecast is constructed as $\ln \left(E_{t}\left(G D P_{t}\right)\right)-\ln \left(E_{t}\left(G D P_{t-1}\right)\right)$. GARCH volatility is the $\sigma_{t+1}$ that comes from estimating (12), using data from 1947q2-2012q2. VIX $X_{t}$ is the Chicago Board Options Exchange Volatility Index closing price on the last day of quarter $t$, from 1990q1-2011q4. BBD policy uncertainty is the Baker, Bloom, and Davis (2012) economic policy uncertainty index for the last month of quarter $t$, from 1985q1-2011q4. $\tilde{U}_{t}$ is nonlinear model uncertainty, measured as the log deviation from trend (eq. 9).

$4.9 \%, \ldots$, between -1 and $-2 \%$, and below $-2 \%$. However, the survey only reports a single probability weight that is averaged across all forecasters.

Since this data does not completely describe the distribution of $y_{t+1}$ beliefs, computing a variance requires some approximation. The most obvious approximation is to assume a discrete distribution. For example, when agents assign a probability to 1-2\% GDP growth, we treat this as if that is the probability placed on the outcome of $1.5 \%$ GDP growth. When the agent says that there is probability $p_{6.5}$ of growth above $6 \%$, we treat this as probability $p_{6.5}$ placed on the outcome $y_{t+1}=6.5 \%$. And if the agent reports probability $p_{-2.5}$ of growth below $-2 \%$, we place probability of $p_{-2.5}$ on $y_{t+1}=-2.5 \%$. Then the expected rate of GDP growth is $\bar{y}=\sum_{m \epsilon M} p_{m} m$ for $M=\{-2.5,-1.5, \ldots, 6.5\}$. Finally, the conditional variance of beliefs about GDP growth are $\operatorname{var}[y \mid \mathcal{I}]=\sum_{m \epsilon M} p_{m}(m-\bar{y})^{2}$.

The resulting conditional variance series is not very informative. It hardly varies (range is $[0.0072,0.0099])$. It does not rise substantially during the financial crisis. In fact, it suggests that uncertainty in 2008 was roughly the same as it was in 2003. The reason this measure does not detect high uncertainty in exteme events is that the growth rates are topand bottom-coded. All extremely bad GDP events are grouped in the bin "growth less than $2 \%$." All the uncertainty was about how bad this recession might be. Instead, what the probability bins reveal is a high probability weight on growth below $2 \%$. Since most of 
the probability is concentrated in one bin, it makes the uncertainty look low. In sum, while using surveys to ask about ex-ante probabilities of GDP events is a promising approach to measuring uncertainty, the available data does not seem useful for our purposes.

\section{Conclusions}

Most approaches to measuring economic uncertainty ignore parameter estimation uncertainty. Sometimes referred to as "rational expectations econometrics," the traditional approach entails estimating a model on the full sample of data and then treating the estimated parameters as truth to infer what the volatility of innovations was in each period in

the past. In equating the volatility with uncertainty, the econometrician is assuming that the uncertain agent knows the true distribution of outcomes at every moment in time and is only uncertain about which outcome will be chosen from this distribution. Assuming such precise knowledge of the economic model rules out most uncertainty and ignores many sources of uncertainty shocks.

We measure uncertainty for an agent who is not endowed with knowledge of the true economic model. Instead, this agent needs to estimate the model of the economy, just like an econometrician. The conditional variance of this agent's forecast, his uncertainty, is much higher and varies more than volatility does. When the agent considers skewed distributions of outcomes, new data or real-time revisions to existing data can change his beliefs about the skewness of the distribution, and thus the probability of extreme events. Small changes in the estimated shape of a probability distribution can increase or decrease the probability of these tail events many-fold. Because these events are so far from the mean outcome, changes in their probability have a large effect on conditional variance, which translates into large shocks to uncertainty. Thus, our message is that it is beliefs about these black swans, extreme events that are never observed, but whose probability is inferred from a forecasting model, that are responsible for much of the shocks to macroeconomic uncertainty. 


\section{References}

Albagli, E., C. Hellwig, and A. Tsyvinski (2013): "A Theory of Asset Pricing Based on Heterogeneous Information," Toulouse School of Economics working paper.

Bachmann, R., and C. Bayer (2012a): "Investment Dispersion and the Business Cycle," University of Aachen working paper.

- (2012b): "Wait-and-See Business Cycles?," University of Aachen working paper.

Bachmann, R., S. Elstner, and E. Sims (2012): "Uncertainty and Economic Activity: Evidence from Business Survey Data," University of Aachen working paper.

Bachmann, R., and G. Moscarini (2012): "Business Cycles and Endogenous Uncertainty," Yale University working paper.

Baker, S., N. Bloom, And S. Davis (2012): "Measuring Economic Policy Uncertainty," Stanford University working paper.

Bansal, R., and I. Shaliastovich (2010): "Confidence Risk and Asset Prices," American Economic Review, 100(2), 537-41.

Barro, R. (2006): "Rare Disasters and Asset Markets in the Twentieth Century," Econometrica, 77(3), 623-685.

BAsu, S., And B. Bundick (2012): "Uncertainty Shocks in a Model of Effective Demand," Boston College working paper.

Bianchi, F., C. Ilut, And M. Schneider (2012): "Ambiguous business cycles and uncertainty shocks," Stanford University working paper.

Bloom, N. (2009): "The Impact of Uncertainty Shocks," Econometrica, 77(3), 623-685.

Bloom, N., M. Floetotto, N. Jaimovich, I. Sapora-Eksten, and S. Terry (2012): "Really Uncertain Business Cycles," NBER working paper 13385.

Born, B., A. Peter, and J. Pfeifer (2011): "Fiscal News and Macroeconomic Volatility," Bonn econ discussion papers, University of Bonn, Germany.

Bruno, V., And H. Shin (2012): "Capital Flows, Cross-Border Banking and Global Liquidity," Princeton University working paper.

Chen, H., W. Dou, and L. Kogan (2013): "Measuring the Dark Matter in Asset Pricing Models," MIT working paper.

Christiano, L., R. Motto, and M. Rostagno (2012): "Risk Shocks," Northwestern University working paper. 
Cogley, T., And T. Sargent (2005): "The Conquest of US Inflation: Learning and Robustness to Model Uncertainty," Review of Economic Dynamics, 8, 528-563.

Collin-Dufresne, P., M. Johannes, and L. Lochstoer (2013): "Parameter Learning in General Equilibrium: The Asset Pricing Implications," Columbia University working paper.

Diether, K., C. Malloy, and A. Scherbina (2002): "Differences of Opinion and the Cross-Section of Stock Returns," Journal of Finance, 57, 2113-2141.

Elliott, G., and A. Timmermann (2008): "Economic Forecasting," Journal of Economic Literature, 46(1), 3-56.

Fajgelbaum, P., E. Schaal, and M. Taschereau-Dumouchel (2013): "Uncertainty Traps," NYU working paper.

Fernandez-Villaverde, J., P. Guerron-Quintana, J. F. Rubio-Ramirez, and M. URIBE (2011): "Risk Matters: The Real Effects of Volatility Shocks," American Economic Review, 101(6), 2530-61.

Fernandez-Villaverde, J., K. Kuester, P. Guerron, and J. Rubio-Ramirez (2012): "Fiscal Volatility Shocks and Economic Activity," University of Pennsylvania working paper.

Hansen, L. (2007): "Beliefs, Doubts and Learning: Valuing Macroeconomic Risk," American Economic Review, 97(2), 1-30.

Johannes, M., L. Lochstoer, and Y. Mou (2011): "Learning About Consumption Dynamics," Columbia University working paper.

Johnson, T. (2004): "Forecast Dispersion and the Cross Section of Expected Returns," Journal of Finance, 59, 1957-1978.

Jurado, K., S. Ludvigson, And S. NG (2013): "Measuring Uncertainty," New York University working paper.

Justiniano, A., And G. Primiceri (2008): "Time-Varying Volatility of Macroeconomic Fluctuations," American Economic Review, 98(3), 604-641.

Nakamura, E., D. Sergeyev, and J. Steinsson (2012): "Growth-Rate and Uncertainty Shocks in Consumption: Cross-Country Evidence," Columbia University working paper.

Nimark, K. (2012): "Man Bites Dog Business Cycles," Working paper. 
Orlik, A., L. VeldKamp, and N. Kozeniauskas (2013): "Micro and Macro Uncertainty Shocks," Working paper.

Pastor, L., And P. Veronesi (2012): "Uncertainty about Government Policy and Stock Prices," Journal of Finance, forthcoming.

Roberts, G., A. Gelman, and W. Gilks (1997): "Weak Convergence and Optimal Scaling of Random Walk Metropolis Algorithms," Annals of Applied Probability, 7(1), 110-120.

Stock, J., And M. Watson (2012): "Disentangling the Channels of the 2007-2009 Recession," NBER working paper 18094.

Straub, L., And R. Ulbricht (2013): "Credit Crunches, Information Failures, and the Persistence of Pessimism," Toulouse School of Economics working paper.

Van Nieuwerburgh, S., and L. Veldkamp (2006): "Learning Asymmetries in Real Business Cycles," Journal of Monetary Economics, 53(4), 753-772.

Wachter, J. (2013): "Can Time-Varying Risk or Rare Disasters Explain Aggregate Stock Market Volatility?," Journal of Finance, 68(3), 987-1035.

\section{A Estimating the model}

In what follows we show how to use Metropolis-Hastings algorithm to generate samples from $p\left(\theta \mid y^{t}\right)$ for each $t=0,1,2, . ., T .{ }^{11}$

The general idea of MCMC methods is to design a Markov chain whose stationary distribution, $\pi$ (with $\pi T=\pi$ where $T$ is a transitional kernel), is the distribution $p$ we are seeking to characterize. In particular, the Metropolis-Hastings sampling algorithm constructs an ergodic Markov chain that satisfies a detailed balance property with respect to $p$ and, therefore, produces the respective approximate samples. The transition kernel of that chain, $T$, is constructed based on sampling from a proposal conditional distribution $q\left(\theta \mid \theta^{(d)}\right)$ where $d$ denotes the number of the sampling step. Specifically, given the $d$-step in the random walk $\theta^{(d)}$ the next-step $\theta^{(d+1)}$ is generated as follows

$$
\theta^{(d+1)}= \begin{cases}\theta^{\prime} & \text { with probability } \alpha\left(\theta^{(d)}, \theta^{\prime}\right)=\min \left(1, \frac{p\left(\theta^{\prime} \mid y^{t}\right)}{p\left(\theta^{(d)} \mid y^{t}\right)} \frac{q\left(\theta^{(d)} \mid \theta^{\prime}\right)}{q\left(\theta^{\prime} \mid \theta^{(d)}\right)}\right) \\ \theta^{(d)} & \text { with probability } 1-\alpha\left(\theta^{(d)}, \theta^{\prime}\right)\end{cases}
$$

where $\theta^{\prime} \sim q\left(\theta \mid \theta^{(d)}\right)$.

\footnotetext{
${ }^{11}$ We drop here the dependence on $\mathcal{M}$ hoping that no confusion arises; the algorithm is applied independently to generate the respective samples under each model.
} 
In our application, the simulation of the parameters is done through simple random walk proposals. In particular, for the means the proposed move is

$$
\mu_{S}^{\prime}=\mu_{S}+\tau_{u} \varepsilon_{S}
$$

where $S \in\{1, \ldots, N\}, \varepsilon_{S} \sim \mathcal{N}(0,1)$.

For the variance $\sigma^{2}$, the proposed move is a multiplicative random walk

$$
\log \sigma^{\prime}=\log \sigma+\tau_{\sigma} \xi_{\sigma}
$$

where $\xi_{\sigma} \sim \mathcal{N}(0,1)$.

In the case of the transition probability matrix, the move is slightly more involved due to the constraint on the sum of rows. We reparameterize each row $\left(q_{i 1}, \ldots, q_{i N}\right)$ as

$$
q_{i j}=\frac{\omega_{i j}}{\sum_{j} \omega_{i j}}, \quad \omega_{i j}>0, \quad j \in\{1, \ldots, N\}
$$

so that the summation constraint does not hinder the random walk. The proposed move on $\omega_{i j}$ is then given by

$$
\log \omega_{i j}^{\prime}=\log \omega_{i j}+\tau_{\omega} \xi_{\omega}
$$

where $\xi_{\omega} \sim \mathcal{N}(0,1)$. Note that this reparametrization requires that we select a prior distribution on $\omega_{i j}$ rather than on $q_{i j}$.

The parameters $\tau_{u}, \tau_{\sigma}$, and $\tau_{\omega}$ can be adjusted to optimize the performance of the sampler. Choosing a proposal with small variance would result in relatively high acceptance rates but with strongly correlated consecutive samples. See Roberts, Gelman, and Gilks (1997) for the results on optimal scaling of the random walk Metropolis algorithm.

Since the proposal is symmetric in all the cases (17), (18), and (19) and since $\varepsilon_{S}, \xi_{\sigma}$, and $\xi_{\omega}$ are drawn independently from one another, we have $q\left(\theta \mid \theta^{\prime}\right)=q\left(\theta^{\prime} \mid \theta\right)$, and the acceptance probability simplifies to $\min \left(1, \frac{p\left(\theta^{\prime} \mid y^{t}\right)}{p\left(\theta^{(d)} \mid y^{t}\right)}\right)$. To compute that acceptance ratio, note that the posterior distribution $p\left(\theta \mid y^{t}\right)$ is given by

$$
p\left(\theta \mid y^{t}\right)=\frac{p\left(y^{t} \mid \theta\right) p(\theta)}{p\left(y^{t}\right)}
$$

where $p\left(y^{t}\right)=\int p\left(y^{t} \mid \theta\right) p(\theta) d \theta$ is the marginal likelihood (or data density).

Continuous state filtering details In turn, the predictive distribution of the data, $p\left(y_{t+1} \mid y^{t}, \theta\right)$ can be obtained as an integral against the filtering distribution

$$
p\left(y_{t+1} \mid y^{t}, \theta\right)=\iint p\left(y_{t+1} \mid s_{t+1}, \theta\right) p\left(s_{t+1} \mid s_{t}, \theta\right) p\left(s_{t} \mid y^{t}, \theta\right) d s_{t} d s_{t+1}
$$


We apply Kalman filtering techniques to obtain the distribution of the filtered values of the hidden state, $p\left(s_{t} \mid y^{t}, \theta\right)$, which will be characterized by the following moments $\widehat{s}_{t} \equiv E\left[s_{t} \mid y^{t-1}, \theta\right]$ and $\operatorname{Var}_{s, t} \equiv E\left[\left(s_{t}-\widehat{s}_{t}\right)\left(s_{t}-\widehat{s}_{t}\right)^{\prime}\right]$. The predictive distribution of the data is then given by the following moments $E\left[y_{t} \mid y^{t-1}, \theta\right]=\widehat{s}_{t}$ and $\operatorname{Var}\left[y_{t} \mid y^{t-1}, \theta\right]=$ $\operatorname{Var}_{s, t}+\sigma^{2}$.

Estimating Prior Beliefs To discipline the priors, we use historical data, i.e. the vintage of the data as of 1968q3 (1947q2-1968q2). We use uniform priors on all the parameters, and estimate respective models using Bayesian techniques described in the Appendix. The mean and standard deviations of the posterior parameter distributions as of 1968q3 become the moments of the prior distributions for respective parameters that will be used in the real-time estimation from 1968q4 onwards. The results for the respective models are reported in the tables below.

To compute volatility in these models, we fix parameters at the estimated means of these prior distributions. Figures 8 and 9 plot the priors and the evolution of parameter beliefs over the sample for the linear-normal and skewed models.

\begin{tabular}{lll}
\hline Parameter & Mean & Stdev \\
\hline$\alpha$ & 2.35 & 0.68 \\
$\rho$ & 0.47 & 0.12 \\
$\sigma^{2}$ & 4.89 & 3.45 \\
$\sigma_{s}^{2}$ & 15.92 & 4.47 \\
\hline
\end{tabular}

Table 3: Moments of the prior distributions in the linear-normal model

\begin{tabular}{lll}
\hline Parameter & Mean & Stdev \\
\hline$c$ & 41.27 & 6.97 \\
$\rho$ & 0.05 & 0.07 \\
$\sigma^{2}$ & 0.02 & 0.01 \\
$\sigma_{s}^{2}$ & 0.005 & 0.007 \\
\hline
\end{tabular}

Table 4: Moments of the prior distributions in the model with skewness.

\section{B Estimated ARCH/GARCH process for GDP}

In this section we present the GARCH models that we estimate to infer volatility proxies for GDP. We estimate volatility using an ARMA model with ARCH or GARCH errors. The estimation procedure is maximum likelihood. We considered several models and chose 

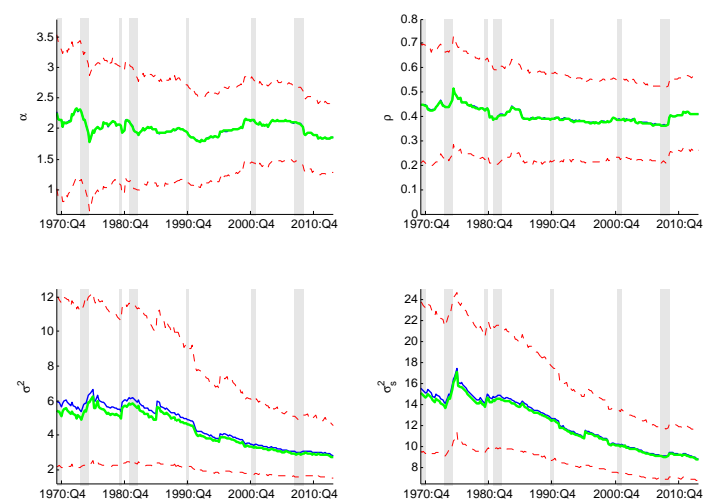

Figure 8: Linear-Normal Model: Posterior Parameter Means, Medians, and 95\%- Credible Sets.
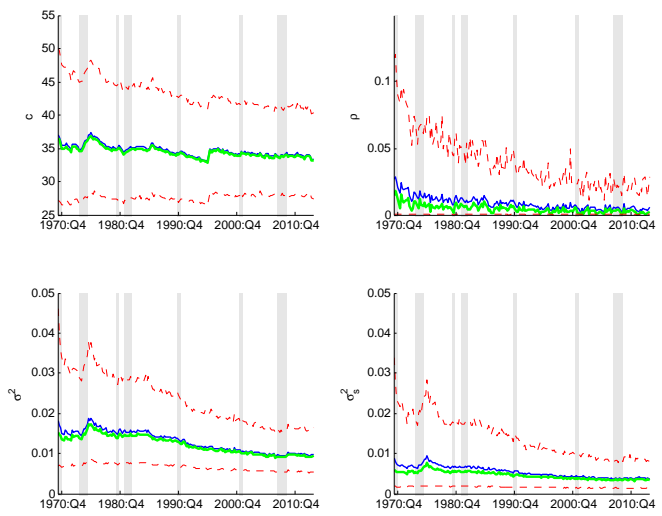

Figure 9: Skewed Model: Posterior Parameter Means, Medians, and 95\%-Credible Sets.

the AR and MA orders based on the significance of additional variables and their effect on the log-likelihood. Similarly, we considered different lags of linear terms for $\epsilon_{t}$ and variances $\sigma_{t}^{2}$ in the GARCH specification and used the significance and effect of additional variables on the log-likelihood to inform the specification choice.

We use quarterly GDP growth rate data for 1947:Q2-2012:Q2. ${ }^{12}$ The subsample of the GDP data which matches the uncertainty data is 1968:Q4 to 2011:Q4. The results of ADF tests indicate that the series for the full sample and matching subsample are stationary.

Best-fitting homoskedastic model Recall that we defined $y_{t+1} \equiv \ln \left(g d p_{t}\right)-\ln \left(g d p_{t-1}\right)$.

\footnotetext{
${ }^{12}$ Data source: Bureau of Economic Analysis (http://www.bea.gov/national/index.htm\#gdp). We are using the seasonally adjusted annual rate for the quarterly percentage change in real GDP. Note that this data is for the actual percentage change, not an approximation. The version of the data is 27 July 2012, downloaded 2 August 2012.
} 
The best-fitting processes is an $\operatorname{ARMA}(1,0)$ :

$$
\Delta g d p_{t+1}=3.25+0.37 \Delta g d p_{t}+\epsilon_{t+1}, \quad \varepsilon_{t+1} \sim \mathcal{N}(0,14.43)
$$

The log-likelihood is -713.27 .

Best-fitting heteroskedastic model For heteroskedastic processes, the best specification is $\operatorname{ARMA}(1,0)$ for growth and $\operatorname{GARCH}(1)$ for variance:

$$
\begin{gathered}
\Delta g d p_{t+1}=3.38+0.41 \Delta g d p_{t}+\epsilon_{t+1}, \quad \varepsilon_{t+1} \sim \mathcal{N}\left(0, \sigma_{t+1}^{2}\right) \\
\sigma_{t+1}^{2}=0.52+0.76 \sigma_{t}^{2}+0.24 \epsilon_{t}^{2}
\end{gathered}
$$

The log-likelihood is -693.90 .

For each sample period of each variable we use Augmented Dickey-Fuller (ADF) tests to check that the series is stationary. This test is based on estimating an AR model for the data. For each sample we use the best-fitting AR model as the basis for the test. Each of the series we use passed the stationarity test. 